\title{
O QUE NOS MOSTRAM PUBLICAÇÕES DE EDUCAÇÃO EM SAÚDE COM FOCO NO ADOLESCENTE?: UM ESTADO DA ARTE EM UMA BASE BIBLIOGRÁFICA LATINO AMERICANA
}

\section{WHAT SHOWS US HEALTH EDUCATION PUBLICATIONS WITH A FOCUS ON TEENAGER?: STATE-OF-THE-ART ON A LATIN AMERICAN BIBLIOGRAPHIC BASE}

\author{
Julio Cesar Bresolin Marinho' ${ }^{1}$, João Alberto da Silva ${ }^{2}$ \\ ${ }^{1}$ Professor do Curso de Ciências da Natureza - Licenciatura da Universidade Federal do Pampa \\ (UNIPAMPA, Campus Uruguaiana) e Doutorando do Programa de Pós-Graduação em Educação em \\ Ciências: Química da Vida e Saúde (Associação ampla entre UFRGS, UFSM e FURG), e-mail: \\ juliomarinho@unipampa.edu.br \\ ${ }^{2}$ Professor da Universidade Federal do Rio Grande-FURG junto ao Programa de Pós-Graduação em \\ Educação em Ciências: Química da Vida e Saúde (Associação ampla entre UFRGS, UFSM e FURG), e- \\ mail: joaosilva@furg.br
}

\section{RESUMO}

O objetivo deste artigo é mapear as temáticas de Educação em Saúde (ES) privilegiadas nas pesquisas publicadas em revistas científicas, disponíveis na SciELO, que enfoquem seus olhares para o público adolescente, bem como as metodologias utilizadas. Realizou-se uma pesquisa qualitativa do tipo Estado da Arte que se estruturou através de busca na SciELO utilizando os descritores "educação em saúde" e "adolescente". Elencamos a técnica da Análise de Conteúdo para organizar a temática dos artigos em duas categorias "identificar e avaliar conhecimentos sobre a saúde dos adolescentes" e "ações educativas de saúde". Em relação as metodologias, evidenciamos que em ambas as categorias está ausente classificações relacionadas à forma de abordagem do problema e aos fins da pesquisa. Já os instrumentos foram descritos em todos os artigos, tendo destaque entrevistas e questionários.

Palavras-chave: Educação em Ciências; Educação em Saúde; Adolescente.

\begin{abstract}
The purpose of this article is to map the topics of Health Education (HE) privileged in the researches published in scientific journals, available on SciELO which focus their attention on the teenager public, as well as the methodologies used. We conducted a qualitative study of the state-of-the-art kind that was structured through search in SciELO using the keywords "health education" and "teenager". We list the content analysis technique to organize the subject of articles in two categories "identify and evaluate knowledge about teenager health" and "health educational activities". Regarding the methodologies we showed that in both categories is absent ratings related to the form of the problem and the purpose of the research approach. The instruments were described in all articles and featured interviews and questionnaires.
\end{abstract}

Key words: Science Education; Health Education; teenager. 


\section{Ensino, Saúde e Ambiente - V10 (1), pp. 123-141, Abril. 2017}

\section{Apresentação do estudo}

O presente artigo insere-se na área de investigação da Educação em Ciências, mais especificamente no campo de conhecimento da Educação em Saúde (ES). Ele busca mapear as temáticas de ES que estão sendo privilegiadas nas pesquisas publicadas em revistas científicas, disponíveis em uma base bibliográfica latino americana que enfocam seus olhares para o público adolescente, bem como as metodologias que estão sendo utilizadas nas pesquisas.

A escolha por investigar o que está sendo publicado no campo da ES relacionado aos adolescentes reside pelo fato da atenção à saúde do adolescente tornarse, no momento atual, uma prioridade em muitos países. Segundo Ruzany (2008), isso decorre da constatação de que a formação do estilo de vida do adolescente é crucial não só para esta etapa da vida, mas também para todo o desenvolvimento da vida adulta e velhice.

Para a construção desse trabalho, partimos do pensamento de Santos, Eliel e Eliel (2006, p. 19), os quais postulam que "a construção de novos conhecimentos e o desenvolvimento da ciência deve se dar a partir dos conhecimentos primeiros e da assimilação das informações que referenciam tais conhecimentos". O que levantamos nessa investigação são os estudos de ES que envolvam os adolescentes para que possamos analisar e compreender as perspectivas que estão sendo exploradas a fim de refletir e avançar nas contribuições nesse campo de estudo.

É pertinente destacarmos que o campo de estudos da ES congrega várias áreas do saber e acaba se configurando como intersetorial e interdisciplinar (MARINHO, 2013; SCHALL; STRUCHINER, 1999). Desse modo, o inventário e análise dos trabalhos do que vem sendo realizados, no que tange ao sujeito adolescente, se faz pertinente em uma dimensão exploratória e abrangente.

\section{Metodologia}

A escolha metodológica se deu por uma abordagem qualitativa do tipo Estado da Arte. Esse tipo de pesquisa se caracteriza como exploratória de caráter bibliográfico, segundo Ferreira (2002, p. 258) traz:

o desafio de mapear e de discutir uma certa produção acadêmica em diferentes campos do conhecimento, tentando responder que aspectos e dimensões vêm sendo destacados e privilegiados em diferentes épocas e lugares, de que formas e em que condições têm sido produzidas certas 


\section{Ensino, Saúde e Ambiente - V10 (1), pp. 123-141, Abril. 2017}

dissertações de mestrado, teses de doutorado, publicações em periódicos e comunicações em anais de congressos e de seminários.

Optamos por realizar a busca em uma das bases bibliográficas latino americanas mais acessadas e prestigiadas, a Scientific Eletronic Library Online SciELO (www.scielo.br), que reúne periódicos online e que permitem acesso livre e gratuito ao texto completo dos artigos. A busca foi realizada durante o mês de julho de 2016, a partir do link "pesquisa de artigos" da SciELO, utilizando a consulta por formulário livre. Para efetuar a pesquisa, realizamos o cruzamento entre os descritores, em língua portuguesa, "educação em saúde" e "adolescente", utilizando o operador booleano and.

Ao realizarmos a busca na SciELO recuperamos 40 artigos, os quais após a realização de uma leitura atenta aos títulos e resumos, nos possibilitou descartar 18 que não apresentaram aderência com a temática investigada. Deste modo, trabalhamos analisando um total de 22 artigos oriundos da SciELO. A análise se deu mediante a técnica da Análise de Conteúdo (BARDIN, 2011). Pela leitura dos títulos e resumos foi possível classificar a temática dos artigos em duas categorias emergentes "identificar e avaliar conhecimentos sobre a saúde dos adolescentes" e "ações educativas de saúde" (B) (Quadro 1).

Quadro 1 - Relação dos artigos que foram analisados e a categoria na qual foram classificados

\begin{tabular}{|c|c|}
\hline \multirow{2}{*}{$\begin{array}{c}\text { Identificar e avaliar } \\
\text { conhecimentos sobre a } \\
\text { saúde dos adolescentes }\end{array}$} & Título do artigo \\
\hline \multirow{15}{*}{$\begin{array}{l}\text { Identificar e avaliar } \\
\text { conhecimentos sobre a } \\
\text { saúde dos adolescentes }\end{array}$} & Prevenção da perda auditiva no contexto escolar frente ao ruído de lazer \\
\hline & $\begin{array}{l}\text { Educação em Saúde com adolescentes: análise da aquisição de } \\
\text { conhecimentos sobre temas de saúde }\end{array}$ \\
\hline & Fatores e motivação para o consumo de bebidas alcoólicas na adolescência \\
\hline & Alimentação e saúde: sentidos atribuídos por adolescentes \\
\hline & $\begin{array}{l}\text { Bebida alcoólica na adolescência: o cuidado-educação como estratégia de } \\
\text { ação da enfermagem }\end{array}$ \\
\hline & $\begin{array}{l}\text { Oficinas sobre sexualidade na adolescência: revelando vozes, desvelando } \\
\text { olhares de estudantes do Ensino Médio }\end{array}$ \\
\hline & Percepções de adolescentes sobre sua sexualidade \\
\hline & $\begin{array}{l}\text { A Educação em Saúde na adolescência: grupos de discussão como } \\
\text { estratégia de pesquisa e cuidado-educação }\end{array}$ \\
\hline & Saberes de adolescentes: estilo de vida e cuidado à saúde \\
\hline & Projeto de vida de um grupo de adolescentes à luz de Paulo Freire \\
\hline & $\begin{array}{l}\text { Estágios de mudança dos pais nas conversas com os filhos sobre } \\
\text { prevenção HIV/AIDS }\end{array}$ \\
\hline & $\begin{array}{l}\text { A saúde no cotidiano de jovens residentes em um bairro popular de Belo } \\
\text { Horizonte, MG, Brasil }\end{array}$ \\
\hline & $\begin{array}{l}\text { Vacinação contra hepatite B em adolescentes residentes em Campinas, São } \\
\text { Paulo, Brasil }\end{array}$ \\
\hline & O significado da educação sexual na relação pais/adolescentes \\
\hline & Percepção de adolescentes sobre a prática de alimentação saudável \\
\hline & $\begin{array}{l}\text { Oficinas educativas como estratégia de promoção da saúde auditiva do } \\
\text { adolescente: estudo exploratório }\end{array}$ \\
\hline
\end{tabular}


Ensino, Saúde e Ambiente - V10 (1), pp. 123-141, Abril. 2017

\begin{tabular}{|c|l|}
\hline \multirow{4}{*}{$\begin{array}{c}\text { Ações educativas de } \\
\text { saúde }\end{array}$} & Reflexões acerca do abuso de drogas e da violência na adolescência \\
\cline { 2 - 3 } & $\begin{array}{l}\text { Avaliação de duas intervenções educativas para a prevenção do Diabetes } \\
\text { Mellitus tipo 2 em adolescentes }\end{array}$ \\
\cline { 2 - 3 } & $\begin{array}{l}\text { Teatro na educação de crianças e adolescentes participantes de ensaio } \\
\text { clínico }\end{array}$ \\
\cline { 2 - 3 } & $\begin{array}{l}\text { Intervenção educacional com base em modelo para aumentar a atividade } \\
\text { física entre adolescentes iranianos }\end{array}$ \\
\hline & $\begin{array}{l}\text { Problematização como estratégia de educação nutricional com } \\
\text { adolescentes obesos }\end{array}$ \\
\hline & $\begin{array}{l}\text { Oficina de autoexame de mamas: uma estratégia para o autoconhecimento } \\
\text { de adolescentes }\end{array}$ \\
\hline
\end{tabular}

Além da análise das temáticas, outro ponto que nos propomos a observar nos artigos residiu na análise das metodologias empregadas. Para essa análise não nos detemos apenas na metodologia apresentada nos resumos dos artigos, pois as mesmas eram muito sucintas. Assim, optamos por realizar a leitura da descrição metodológica presente no corpo do texto.

Optamos por organizar os critérios de enquadramento metodológico baseado nas referencias propostas por Gerhardt e Silveira (2009) e Prodanov e Freitas (2013). Desse modo, analisamos as metodologias no que se refere a quatro aspectos: quanto a abordagem do problema; quanto aos fins de pesquisa (objetivos); quanto aos procedimentos/técnicas utilizadas e quanto aos tipos de instrumentos.

\section{Análise referente à leitura dos títulos, resumos e metodologias dos artigos}

A primeira inferência que podemos realizar sobre a análise realizada é que dos 22 artigos que foram selecionados no estudo, 15 deles (Figura 1) se encontram em periódicos especializados da área da Enfermagem. Essa evidência se alia a estudos anteriores (MARINHO, 2013; MARINHO, TAUCHEN, SILVA, 2012), nos quais os autores concluíram que o maior percentual de artigos publicados na SciELO, contendo os descritores "educação em saúde" e "escola", foram os produzidos por profissionais da área da Enfermagem (95 de um total de 151 artigos). Outros três artigos encontramse dispersos em revistas genéricas da área da saúde (Cadernos de Saúde Pública; Ciência \& Saúde Coletiva; Revista de Saúde Pública) e os restantes encontram-se em revistas especializadas da medicina e da fonoaudiologia (Jornal de Pediatria, Revista Brasileira de Epidemiologia e Audiology - Communication Research). Salientamos também, que os autores dos três artigos publicados nas revistas genéricas da área da saúde são enfermeiros e/ou pesquisadores que atuam em Escolas de Enfermagem nas universidades do país. 
Figura 1: Distribuição dos artigos encontrados no SciELO por periódicos

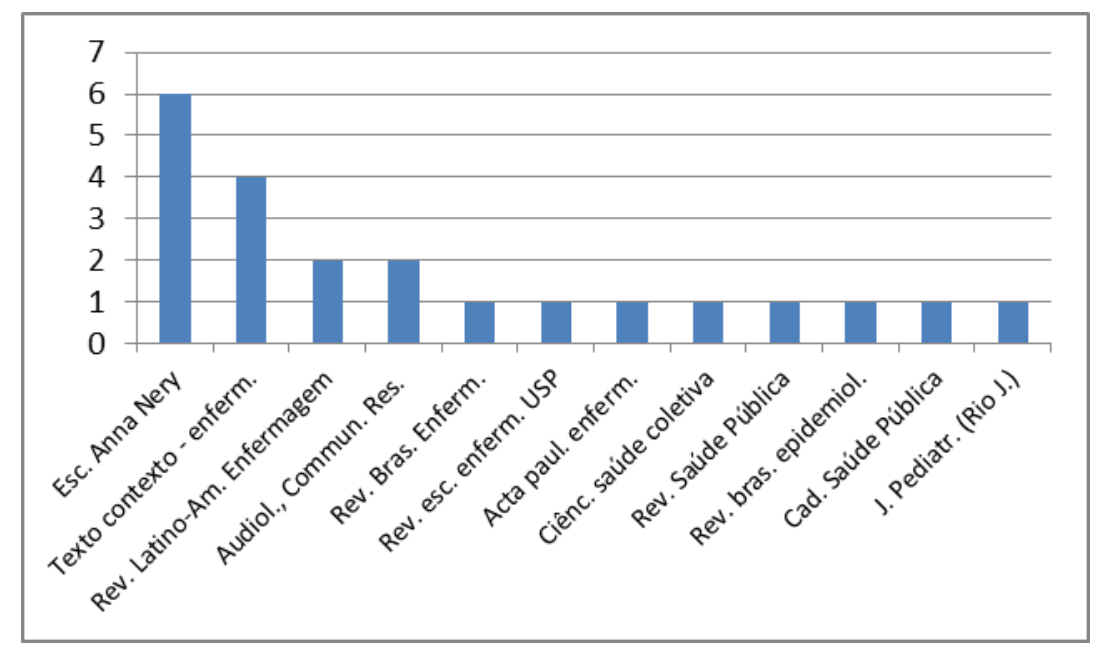

Fonte: Elaborada pelos autores.

Após a realização da leitura dos títulos e resumos dos 22 artigos selecionados, estes foram classificados em duas categorias: "identificar e avaliar conhecimentos sobre a saúde dos adolescentes" (A), no qual foram enquadrados 15 artigos e "ações educativas de saúde" (B), que aglutinou 7. Apresentaremos agora os resultados obtidos nessas categorias da seguinte forma: primeiramente apresentamos a descrição da categoria A, após a discussão referente às temáticas dos artigos dessa categoria, e por fim a discussão no que tange as escolhas metodológicas dos autores. Salientamos que a mesma organização foi realizada para apresentação dos resultados da categoria B.

\section{(A) Identificar e avaliar conhecimentos sobre a saúde dos adolescentes}

Nessa categoria apresentamos os artigos que tratam sobre aspectos relacionados com a identificação e avaliação de conhecimentos sobre a saúde dos adolescentes. Observamos que as questões de sexualidade, alimentação e bebidas alcoólicas são as privilegiadas em relação a saúde dos adolescentes. Outras questões como saúde bucal; prevenção ao uso de drogas; uso de estéreos pessoais; cobertura vacinal contra hepatite $\mathrm{B}$; o conhecimento do projeto de vida de adolescentes, também são exploradas pelos autores dos artigos. Discussões referentes a conhecer as concepções dos adolescentes sobre saúde e como estas se articulam com as suas práticas de cuidado, e conhecer os modos de vida juvenis apreendendo os significados e sentidos da saúde aparecem na análise. 


\section{Ensino, Saúde e Ambiente - V10 (1), pp. 123-141, Abril. 2017}

\section{A.1 - Análise das temáticas dos estudos}

Os artigos classificados na categoria "identificar e avaliar conhecimentos sobre a saúde dos adolescentes" apresentavam enfoques variados sobre saúde, destacando-se a questão da sexualidade, da alimentação e das bebidas alcoólicas. Em relação aos artigos que abordam a questão da sexualidade na adolescência apresentamos o estudo de Soares, Amaral, Silva e Silva (2008), o qual procurou compreender como adolescentes de uma escola estadual de Minas Gerais vivem e exercitam sua sexualidade. Tais autores evidenciaram que o conceito de sexualidade limita-se às relações sexuais entre duas pessoas de sexo oposto; que os alunos enfatizaram o risco de uma gravidez indesejada e reconheceram a importância do uso de métodos contraceptivos. O estudo de Freitas e Dias (2010) se aproxima do de Soares et al. (2008), pois tiveram como objetivo conhecer as percepções dos adolescentes sobre o desenvolvimento da sua sexualidade. Freitas e Dias (2010, p. 356) evidenciaram que:

\footnotetext{
Os adolescentes revelaram que seus interesses em relação à sexualidade estão ligados à afetividade e à busca de um parceiro, ou seja, à procura do objeto sexual, ainda que relatem preocupação com a prevenção de DST e gravidez na adolescência. Eles ressaltaram, ainda, os conflitos que vivenciam nessa fase, como um momento de transição, marcado pelas conquistas próprias da adolescência.
}

Outros dois artigos correlatos com a questão da sexualidade trazem a figura dos pais de adolescentes para a investigação. O estudo de Barbosa, Costa e Vieira (2008) procurou conhecer o estágio de mudança do comportamento dos pais em relação à comunicação com os filhos adolescentes sobre sexo/sexualidade e medidas preventivas de HIV/AIDS. Tais autores observaram que a maioria dos pais relatou que conversam ou têm interesse em conversar com os filhos sobre a temática, apesar de alguns terem evidenciado dificuldades para isso, havendo necessidade de maior esclarecimento sobre medidas preventivas de HIV/AIDS ou gravidez indesejada. Já no artigo de Jesus (1999), a autora procurou compreender o típico da ação de pais e adolescentes frente à educação para a vida sexual e verificou a necessidade de implementação do diálogo sobre a vida sexual na relação pais/adolescentes, com vistas a uma iniciação sexual segura e feliz.

A questão da alimentação dos adolescentes é evidenciada na pesquisa de Silva, Teixeira e Ferreira (2010) e Silva, Frazão, Osório e Vasconselos (2015). O trabalho de Silva et al. (2010) procurou: identificar o saber de adolescentes sobre uma alimentação saudável; analisar os alimentos que compõem suas refeições; discutir este consumo à 


\section{Ensino, Saúde e Ambiente - V10 (1), pp. 123-141, Abril. 2017}

luz de uma alimentação saudável e programar ações de educação em saúde sobre o tema. Este estudo nos apresenta um resultado interessante, que apesar dos adolescentes saberem sobre os hábitos saudáveis de alimentação, a maioria declarou não os seguir. $\mathrm{O}$ artigo de Silva et al. (2015) procurou analisar a percepção sobre a prática de alimentação saudável de adolescentes em uma escola de Pernambuco e constataram em consonância com o estudo de Silva et al. (2010) que os adolescentes entrevistados, apesar de terem conhecimento sobre alimentação saudável, nem sempre o põem em prática, devido à multiplicidade de fatores que interferem em suas escolhas alimentares.

Um tema que merece atenção, quando se trata da questão da Educação em Saúde com adolescentes, é no que se refere às bebidas alcoólicas. Mendes, Teixeira e Ferreira (2010) procuram identificar o significado atribuído pelos adolescentes ao consumo de bebidas alcoólicas; caracterizar a quantidade e os principais locais de consumo dessas bebidas; e detectar fatores de risco e/ou tendência para este consumo. No artigo de Neves, Teixeira e Ferreira (2015) o objetivo consistiu em identificar os fatores que influenciam os adolescentes ao consumo de bebidas alcoólicas, suas motivações e seus saberes sobre esta prática. Ambos os trabalhos possuem objetivos próximos, pois procuram identificar o que influencia os adolescentes a consumirem bebidas alcoólicas e chegam a resultados interessantes.

Em relação aos resultados das investigações, Mendes et al. (2010) visualizam que o maior consumo de bebidas alcoólicas pelos adolescentes ocorre em festas e comemorações, evidenciando o apelo social do álcool, que representa um facilitador para a interação, socialização e permissividade de atitudes e pensamentos não aceitos socialmente. Já Neves et al. (2015) constatam que a cerveja é a principal bebida consumida pelos adolescentes de seu estudo e a diversão, a companhia de amigos e a fuga da realidade são as principais finalidades para o consumo. Nessa investigação evidencia-se que os adolescentes apesar de conhecerem alguns dos riscos, consomem as bebidas alcoólicas, geralmente, em grupos.

O estudo de Viero, Farias, Ferraz, Simões, Martins e Cereta (2015) buscou analisar a aquisição de conhecimentos sobre temas variados como: saúde bucal; prevenção ao uso de drogas e sexualidade, junto a adolescentes matriculados na rede pública de ensino do Sul de Santa Catarina. Após o desenvolvimento do estudo, os autores visualizaram o aumento de conhecimento dos adolescentes nas temáticas sobre prevenção de drogas e sexualidade, fato que não se configurou na temática saúde bucal. Na mesma linha de pensamento, o estudo de Ferreira, Alvin, Teixeira e Veloso (2007) 


\section{Ensino, Saúde e Ambiente - V10 (1), pp. 123-141, Abril. 2017}

procurou conhecer as concepções dos adolescentes sobre saúde e como estas se articulam com as suas práticas de cuidado, na especificidade do processo de adolescer. Já Horta e Sena (2011) analisaram os modos de vida juvenis apreendendo os significados e sentidos da saúde em seu cotidiano. Todos esses três estudos procuraram investigar aspectos relacionados com a concepção de saúde dos adolescentes e possíveis implicações para seu viver.

Dois aspectos específicos de saúde foram abordados nos estudos de Santana, Alvarenga, Cruz, Quadros e Jacob-Corteletti (2016) e Fancisco, Donalisio, Gabriel e Barros (2015). O artigo de Santana et al. (2016) procurou verificar os hábitos e atitudes de jovens em idade escolar com relação ao uso de estéreos pessoais e avaliar o nível de conhecimento sobre os efeitos nocivos da exposição à música amplificada na audição, antes e após uma palestra educativa, dessa maneira evidenciaram que grande parte dos escolares possuía conhecimento prévio a respeito dos prejuízos da música amplificada, para a audição e, ainda assim, alguns referiram hábitos inadequados. O objetivo da investigação de Francisco et al. (2015) residiu em avaliar a cobertura vacinal contra hepatite B em adolescentes e identificar os fatores associados e motivos da não adesão, para isso realizaram um estudo transversal de base populacional, pelo qual foi possível evidenciar que os principais motivos para a não adesão eram a falta de orientação e não considerar a vacina necessária. Já condições socioeconômicas, comportamentos e condições de saúde não restringiram o acesso à vacinação, mas a cobertura esteve abaixo da meta estabelecida pelo Ministério da Saúde.

Ferreira (2006) realiza uma pesquisa com adolescentes para investigar as suas representações de corpo, articuladas aos cuidados à saúde e evidenciou que:

\footnotetext{
os adolescentes fizeram reflexões importantes sobre suas experiências de vida, e verbalizaram suas angústias, seus anseios e suas dúvidas e, inusitadamente, passaram a questionar a pesquisadora sobre os pontos focos da discussão, sabedores de que a mesma era profissional da saúde. Deixaram claro, portanto, a contrapartida que queriam pela participação no processo da pesquisa (FERREIRA, 2006, p. 210).
}

Para finalizar essa categoria apresentamos o estudo de Cardoso e Cocco (2003), as quais, na perspectiva de Paulo Freire, se propuseram a conhecer o projeto de vida de um grupo de adolescentes de uma Unidade Básica de Saúde em Marília, São Paulo. Essas constataram que os adolescentes têm um projeto de vida, apesar das 


\section{Ensino, Saúde e Ambiente - V10 (1), pp. 123-141, Abril. 2017}

dificuldades próprias das condições socioeconômicas a que pertencem, fato por eles percebido.

\section{A.2 - Análise das metodologias utilizadas nos estudos}

Nos trabalhos classificados na categoria "identificar e avaliar conhecimentos sobre a saúde dos adolescentes" o primeiro mapeamento que apresentamos refere-se à escolha metodológica relacionada à forma de abordagem do problema (quanti ou qualitativa). Em relação a esse primeiro olhar, encontramos 9 estudos com abordagem qualitativa, um com abordagem quali-quantitativa, nenhum quantitativo e 5 que não mencionaram o tipo de abordagem (Tabela 1).

No que se trata da abordagem metodológica quanto aos fins da pesquisa (exploratória, descritiva e explicativa), podemos visualizar pouca menção a essa descrição nos artigos, visto que $12(\mathrm{n}=15)$ artigos dessa categoria não informaram tal descrição em suas metodologias. Os três trabalhos que realizaram menção classificaram seus estudos em: descritivo, descritivo/exploratório e exploratório/interpretativo (Tabela $1)$.

Assim como na descrição da abordagem metodológica quanto aos fins da pesquisa, o item "metodologia quanto aos procedimentos/técnicas" também apresenta pouco detalhamento nos artigos e, $8(53,33 \%)$, não informam o enquadramento de seu estudo. Dos trabalhos que apresentam essa descrição, três realizaram uma pesquisa convergente assistencial, dois uma pesquisa de campo, um artigo apresenta um estudo transversal de base populacional e outro utiliza o Método Criativo Sensível (MCS) (Tabela 1).

Em se tratando da metodologia, quanto aos tipos de instrumentos, todos os artigos apresentaram os instrumentos utilizados em suas investigações, alguns deles inclusive optando pela utilização de mais de um (Tabela 1). As entrevistas foram destaque nessa categoria, aparecendo em 8 trabalhos $(38,10 \%)$, seguida dos questionários - aposta de três estudos (14,29\%). Grupo focal e técnica da fotolinguagem foram a escolha de dois trabalhos. Já os instrumentos: discussões em grupos, técnicas verbais da livre-associação de ideias, observação participante, dinâmicas de criatividade e sensibilidade, oficinas lúdico pedagógicas e desenvolvimento de ações de Educação em Saúde, são mencionados cada um uma única vez. 


\section{Ensino, Saúde e Ambiente - V10 (1), pp. 123-141, Abril. 2017}

Tabela 1: Síntese das quatro classificações em relação à metodologia dos artigos da categoria $\mathrm{A}$

\begin{tabular}{|c|c|c|c|}
\hline Classificação & Descrição & $\mathbf{N}$ & $\%$ \\
\hline \multirow{4}{*}{$\begin{array}{l}\text { Metodologia quanto à forma de } \\
\text { abordagem do problema }\end{array}$} & Qualitativa & 9 & 60,00 \\
\hline & Quali-quantitativa & 1 & 6,67 \\
\hline & Não informada & 5 & 33,33 \\
\hline & Total & 15 & 100,00 \\
\hline \multirow{5}{*}{$\begin{array}{l}\text { Metodologia quanto aos fins de } \\
\text { pesquisa }\end{array}$} & Descritiva & 1 & 6,67 \\
\hline & Descritiva/exploratória & 1 & 6,67 \\
\hline & Exploratória/interpretativa & 1 & 6,67 \\
\hline & Não informada & 12 & 80,00 \\
\hline & Total & 15 & 100,00 \\
\hline \multirow{6}{*}{$\begin{array}{l}\text { Metodologia quanto aos } \\
\text { procedimentos/técnicas }\end{array}$} & Pesquisa convergente assistencial & 3 & 20,00 \\
\hline & Pesquisa de campo & 2 & 13,33 \\
\hline & Estudo transversal de base populacional & 1 & 6,67 \\
\hline & Método Criativo Sensível (MCS) & 1 & 6,67 \\
\hline & Não informada & 8 & 53,33 \\
\hline & Total & 15 & 100,00 \\
\hline \multirow{11}{*}{$\begin{array}{l}\text { Metodologia quanto aos tipos } \\
\text { de instrumento }\end{array}$} & Entrevista & 8 & 38,10 \\
\hline & Questionário & 3 & 14,29 \\
\hline & Grupo focal & 2 & 9,52 \\
\hline & Técnica da foto-linguagem & 2 & 9,52 \\
\hline & Discussões em grupo & 1 & 4,76 \\
\hline & Técnicas verbais da livre-associação de ideias & 1 & 4,76 \\
\hline & Observação participante & 1 & 4,76 \\
\hline & Dinâmicas de criatividade e sensibilidade & 1 & 4,76 \\
\hline & Oficinas lúdico pedagógicas & 1 & 4,76 \\
\hline & $\begin{array}{l}\text { Desenvolvimento de ações de Educação em } \\
\text { Saúde }\end{array}$ & 1 & 4,76 \\
\hline & Total & $21^{1}$ & 100,00 \\
\hline
\end{tabular}

\section{(B) Ações educativas de saúde}

Nessa categoria aglutinamos 7 artigos que objetivaram desenvolver alguma ação educativa sobre saúde, tendo os adolescentes como público alvo. As temáticas que acabam ganhando destaque nas investigações são: saúde auditiva e exposição a ruídos; uso abusivo de drogas e comportamentos violentos; educação nutricional; prevenção do diabetes; vacinação; programa para melhorar a atividade física; oficina de autoexame de mamas.

\section{B.1 - Análise das temáticas dos estudos}

Inicialmente apresentamos o trabalho de Lacerda, Soares Gonçalves e Lopes (2013) os quais desenvolveram e avaliaram oficinas educativas sobre saúde auditiva e exposição a ruídos de 91 adolescentes escolares da rede pública de Ensino Médio. Tais pesquisadores evidenciaram que após as oficinas, por meio de questionários aplicados,

\footnotetext{
${ }^{1}$ Nessa classificação o total foi maior que o número de artigos (15), pelo fato de alguns estudos possuírem mais de um tipo de instrumento.
} 


\section{Ensino, Saúde e Ambiente - V10 (1), pp. 123-141, Abril. 2017}

foram observadas mudanças na compreensão dos jovens, os quais passaram a considerar o ruído como algo ruim e danoso à saúde.

Para a investigação de Silva, Dias, Vieira e Pinheiro (2010) realizou-se ações de Educação em Saúde visando à reflexão crítica de 23 adolescentes sobre o uso abusivo de drogas e consequentes comportamentos violentos. Neste estudo foi possível observar que os adolescentes experimentam as drogas por desinformação, curiosidade e fácil acesso. Silva et al. (2010) apostam que as estratégias de Educação em Saúde direcionadas aos adolescentes contribuem para um padrão de vida mais saudável, pois facilita a identificação dos fatores de riscos e têm a finalidade de reduzir a vulnerabilidade desses adolescentes.

As questões relacionadas a alimentação emergem em dois estudos. Rodrigues e Boog (2006) procuraram avaliar a intervenção de educação nutricional, empregando o método da problematização com 22 adolescentes obesos. Os autores puderam concluir que a intervenção foi eficaz para ajudar os adolescentes a compreenderem sua história de vida e determinantes do comportamento alimentar, efetivarem mudanças na sua alimentação espontaneamente, conscientizarem-se das possibilidades de perpetuação da mudança das práticas alimentares e exercerem com autonomia o papel de sujeitos no cuidado à saúde. No estudo de Silva, Zanetti, Forti, Freitas, Hissa e Damasceno (2011), os autores se propuseram a avaliar duas intervenções educativas para a prevenção do Diabetes Mellitus tipo 2 em adolescentes de risco e constataram que as duas intervenções educativas podem ser utilizadas nas escolas para a prevenção do diabetes.

Gazzinelli, Souza, Araújo, Costa, Soares e Maia (2012) procuraram analisar os efeitos de uma intervenção pedagógica na aprendizagem de crianças e adolescentes participantes de pesquisa clínica. O estudo parte de um conjunto de estudos envolvidos no teste de uma vacina contra ancilostomíase e evidenciaram que houve um aumento do conhecimento sobre sinais e sintomas, susceptibilidade à reinfecção e modo de contágio da verminose após a intervenção educativa. No seu estudo, Sanaeinasab, Saffari, Pakpour, Nazeri e Piper (2012), procuraram avaliar um programa educacional com base no modelo de promoção da saúde (MPS) e nos estágios de mudança para melhorar a atividade física $(\mathrm{AF})$ entre adolescentes iranianos e chegaram à conclusão de que intervenções educacionais com base nos estágios de mudança podem ter implicações importantes na melhora da AF entre adolescentes em mais componentes do MPS.

Por fim, Grego, Ohara, Pereira e Brêtas (2011) verificaram a repercussão do conhecimento transmitido por meio da oficina de autoexame de mamas e identificar a 


\section{Ensino, Saúde e Ambiente - V10 (1), pp. 123-141, Abril. 2017}

multiplicação de informações pelas participantes adolescentes. Os autores evidenciaram que a oficina auxilia a elaboração do conceito de si pelas adolescentes, de seu corpo, informa sobre o câncer de mama e os benefícios da adoção de práticas e atitudes saudáveis em seu cotidiano, empregando a técnica do autoexame de mamas como instrumento pedagógico.

\section{B.2 - Análise das metodologias utilizadas nos estudos}

Nas metodologias empregadas nos trabalhos classificados na categoria "ações educativas de saúde", no que refere-se à escolha metodológica relacionada a forma de abordagem do problema (quanti ou qualitativa) observamos que dois estudos são qualitativos, dois quantitativos e três deles não mencionam o tipo de abordagem (Tabela 2).

Em relação a abordagem metodológica quanto aos fins da pesquisa (exploratória, descritiva e explicativa), observamos que em nenhum dos 7 artigos $(100,00 \%)$ é feita menção a essa descrição (Tabela 2). Já na classificação da metodologia quanto aos procedimentos/técnicas em apenas um artigo não é informado o enquadramento de seu estudo. Dos trabalhos que apresentam essa descrição, dois são descritos como quase-experimental; um trata-se de uma pesquisa ação; outro é um estudo de intervenção; um é do tipo Survey e outro configura-se como um estudo randomizado por agrupamento (Tabela 2).

A classificação da metodologia quanto aos tipos de instrumentos, é apresentada em todos os artigos, alguns deles, por vezes, optando pela utilização de mais de um instrumento (Tabela 2). Os questionários foram destaque nessa categoria, aparecendo em 5 trabalhos $(50,00 \%)$, seguido de entrevista, grupo focal, observação, formulário e teatro do oprimido, todos aparecendo em uma única vez nos artigos.

Tabela 2: Síntese das quatro classificações em relação à metodologia dos artigos da categoria $B$

\begin{tabular}{c|l|c|c}
\hline Classificação & \multicolumn{1}{|c|}{ Descrição } & $\mathbf{N}$ & $\mathbf{\%}$ \\
\hline \multirow{3}{*}{$\begin{array}{c}\text { Metodologia quanto à forma de } \\
\text { abordagem do problema }\end{array}$} & Qualitativa & 2 & 28,57 \\
\cline { 2 - 4 } & Quantitativa & 2 & 28,57 \\
\cline { 2 - 4 } & Não informada & 3 & 42,86 \\
\cline { 2 - 4 } & \multicolumn{1}{|c|}{ Total } & 7 & 100,00 \\
\hline \multirow{2}{*}{$\begin{array}{c}\text { Metodologia quanto aos fins de pesquisa } \\
\text { Metodologia quanto aos } \\
\text { procedimentos/técnicas }\end{array}$} & Não informada & 7 & 100,00 \\
\cline { 2 - 4 } & Total & 7 & 100,00 \\
\hline & Pesquisa ação & 1 & 28,57 \\
\cline { 2 - 4 } & Estudo de intervenção & 1 & 14,29 \\
\cline { 2 - 4 } & $\begin{array}{l}\text { Estudo randomizado por } \\
\text { agrupamento }\end{array}$ & 1 & 14,29 \\
\hline
\end{tabular}


Ensino, Saúde e Ambiente - V10 (1), pp. 123-141, Abril. 2017

\begin{tabular}{l|l|c|c}
\hline \multirow{4}{*}{\begin{tabular}{c|l|l} 
Metodologia quanto aos tipos de \\
instrumento
\end{tabular}} & Survey & 1 & 14,29 \\
\cline { 2 - 4 } & Não informada & 1 & 14,29 \\
\cline { 2 - 4 } & \multicolumn{1}{|c|}{ Total } & 7 & 100,00 \\
\hline & Questionário & 5 & 50,00 \\
\cline { 2 - 4 } & Entrevista & 1 & 10,00 \\
\cline { 2 - 4 } & Grupo focal & 1 & 10,00 \\
\cline { 2 - 4 } & Observação & 1 & 10,00 \\
\cline { 2 - 4 } & Formulário & 1 & 10,00 \\
\cline { 2 - 4 } & Teatro do oprimido & 1 & 10,00 \\
\cline { 2 - 4 } & Total & $10^{2}$ & 100,00 \\
\hline
\end{tabular}

\section{Considerações finais}

Com o desenvolvimento do estudo evidenciamos que a maioria dos artigos analisados encontrava-se em periódicos especializados da área da Enfermagem, notando uma ausência de periódicos e pesquisadores da área de Educação em Ciências. Os artigos puderam ser classificados em duas categorias: identificar e avaliar conhecimentos sobre a saúde dos adolescentes (A) e ações educativas de saúde (B).

Pela análise dos artigos que integraram a categoria A podemos inferir que os temas privilegiados residiam em: sexualidade, alimentação e bebidas alcoólicas. Acreditamos que o maior destaque em torno dessas temáticas ocorra em virtude do maior impacto/maior relação que acabam por ocasionar no sujeito adolescente. Nesse contexto, é importante lembrarmos que a adolescência se configura como "um momento de crises, transformações, mudanças de ordem física, cognitiva, psíquica e social, proporciona ao adolescente novas formas de se relacionar com seu mundo interno e externo" (CRUZ, SILVA e TEIXEIRA, 2015, p. 111). Gonçalves (2015) expõem, que nesse período, se abandona a identidade infantil e busca-se uma nova identidade com características adultas, que busca uma nova forma de ser.

Em um cenário de transformações e crises, no qual se busca uma identidade, o adolescente inicia sua vida sexual, o grupo de amigos acaba por ter um papel importante em suas ações e escolhas. Acreditamos que por essas características, tais temáticas (sexualidade, alimentação e bebidas alcoólicas) acabam por ser privilegiadas nas investigações dos autores. Outra possibilidade existente para o destaque dessas temáticas, pode residir pela permanência de características do paradigma biomédico de saúde, visto que tais temáticas aproximam a saúde das questões orgânicas e do corpo. Outros campos da Educação Saúde podem ter menor destaque por que pouco interessam

\footnotetext{
${ }^{2}$ Nessa classificação o total foi maior que o número de artigos (7), pelo fato de alguns estudos possuírem mais de um tipo de instrumento.
} 


\section{Ensino, Saúde e Ambiente - V10 (1), pp. 123-141, Abril. 2017}

para a cura de doenças, intervenções ou veiculação de informação. Dessa maneira, as temáticas mais desenvolvidas identificam uma certa episteme da área.

Da análise das temáticas dos artigos da categoria "identificar e avaliar conhecimentos sobre a saúde dos adolescentes" emergem resultados interessantes que nos possibilitam reflexão. Nos artigos sobre alimentação, os autores constataram que apesar dos adolescentes saberem/terem conhecimento sobre os hábitos saudáveis, a maioria declarou não os seguir. Fato evidenciado também nos artigos que abordaram a ideia do consumo de bebidas alcoólicas e no que investigou os prejuízos da música amplificada para a audição. O que esses resultados nos permitem inferir? Com eles, podemos evidenciar que em muitas situações o sujeito adolescente tem conhecimento do que traz benefícios/malefícios para sua saúde, e em dadas ocasiões opta por fazer aquilo que não é o melhor para consigo.

Mas, por qual motivo ter conhecimento acaba por não ser suficiente? Nossa hipótese é que, na maior parte das vezes, o sujeito aprende "movido" pelo dever (devese agir de tal forma), por meio de técnicas muito coercitivas, sem explicar o porquê de determinada ação. Dessa forma, em determinadas situações, os adolescentes podem agir de forma contrária ao que lhes foi passado. Tognetta e Assis (2006, p. 53) apontam que "saber qual o dever a cumprir não impede um sujeito de agir mal". O "agir mal", no que se refere a sua saúde, reside em escolhas que coloquem em risco sua integridade física e psíquica.

A categoria B aglutinou artigos que realizaram ações educativas sobre saúde com adolescentes. As temáticas que emergiram nessa categoria foram diversas, não havendo hegemonia de nenhum tema em especial. O que evidenciamos é que nas diversas intervenções realizadas os autores observaram que ocorreram mudanças no entendimento dos jovens, como podemos visualizar na constatação de um dos trabalhos sobre ancilostomíase, no qual os autores evidenciaram um aumento do conhecimento sobre sinais e sintomas, susceptibilidade à reinfecção e modo de contágio da verminose após a intervenção educativa desenvolvida. Nesse contexto, o que podemos inferir, baseados no que foi constatado na categoria $\mathrm{A}$, é que o aumento de conhecimento do adolescente não garante uma ação efetiva em prol de sua saúde.

Para a realização de ações educativas de saúde, no Ensino de Ciências, com adolescentes estamos apostando na utilização de dilemas morais (MARINHO, SILVA e CAETANO, 2017). O trabalho com dilemas possibilita uma participação ativa dos adolescentes, "trazendo à tona os seus conflitos, as suas frustrações e as suas aspirações 
- assumindo-os, refletindo sobre eles e discutindo com os outros -, buscando solucionálos de maneira construtiva" (GONÇALVES, 2015, p. 115). Acreditamos assim, que o trabalho por meio dos dilemas morais pode contribuir com o desenvolvimento de uma prática de Educação em Saúde mais autônoma, com maior significado para o sujeito adolescente.

No que tange ao mapeamento em relação às metodologias, em ambas as categorias, visualizamos a ausência de classificações em um número considerável de artigos no que se refere à escolha metodológica relacionada: à forma de abordagem do problema e aos fins da pesquisa. A classificação quanto aos procedimentos/técnicas não aparece em muitos artigos da categoria $\mathrm{A}$, mas está mencionada em praticamente todos os artigos da categoria B. Em se tratando da metodologia quanto aos tipos de instrumentos, todos os artigos - em ambas as categorias - descreveram os instrumentos utilizados em suas investigações, em alguns deles inclusive optando pela utilização de mais de um instrumento. Dentre os instrumentos escolhidos pelos autores, constatamos que nos trabalhos da categoria "identificar e avaliar conhecimentos sobre a saúde dos adolescentes" as entrevistas foram a aposta predominante, já na categoria "ações educativas de saúde" os questionários se mostraram mais expressivos.

\section{Referências}

BARBOSA, S.M.; COSTA, P.N.P.; VIEIRA, N.F.C. Stages of change in parents' discussions with their children about HIV/Aids prevention. Rev. Latino-Am.

Enfermagem, Ribeirão Preto, v. 16, n. 6, p. 1019-1024, dez. 2008. Disponível em: $<$ http://www.scielo.br/scielo.php?script=sci_arttext\&pid=S0104-11692008000 600013\&lng=en\&nrm=iso >. Acesso em: 20 jul. 2016.

BARDIN, L. Análise de conteúdo. São Paulo: Edições70, 2011.

CARDOSO, C.P.; COCCO, M.I.M. Projeto de vida de um grupo de adolescentes à luz de Paulo Freire. Rev. Latino-Am. Enfermagem, Ribeirão Preto, v. 11, n. 6, p. 778785, dez. 2003. Disponível em: <http://www.scielo.br/scielo.php?script=sci_arttext\& pid=S0104-11692003000600012\&lng=pt\&nrm=iso > . Acesso em: 21 jul. 2016.

CRUZ, L.A. N.; SILVA, I.A.; TEIXEIRA, P.S. A vulnerabilidade do adolescente frente ao consumo de bebidas alcoólicas. In: MARTINS, R.A.; CRUZ, L.A.N.

Desenvolvimento sócio moral e condutas de risco em adolescentes. Campinas: Mercado de Letras, 2015. p. 101-115.

FERREIRA, N.S.A. As pesquisas denominadas "estado da arte". Educ. Soc., Campinas, v. 23, n. 79, p. 257-272, ago. 2002. Disponível em: <http://www.scielo.br/scielo.php? script=sci_arttext\&pid=S0101-73302002000300013\&lng=en\&nrm=iso $>$. Acesso em: 06 ago. 2016. 
FERREIRA, M.A. A educação em saúde na adolescência: grupos de discussão como estratégia de pesquisa e cuidado-educação. Texto contexto - enferm., Florianópolis, v. 15, n. 2, p. 205-211, jun. 2006. Disponível em: <http://www.scielo.br/scielo.php?script= sci_arttext\&pid=S0104-07072006000200003\&lng=pt\&nrm=iso>. Acesso em: 21 jul. 2016.

FERREIRA, M.A.; ALVIM, N.A.T.; TEIXEIRA, M.L.O.; VELOSO, R.C. Saberes de adolescentes: estilo de vida e cuidado à saúde. Texto contexto enferm., Florianópolis, v. 16, n. 2, p. 217-224, jun. 2007. Disponível em: <http://www.scielo.br/scielo.php? script=sci_arttext\&pid=S0104-07072007000200002\&lng=pt\&nrm=iso $>$. Acessos em: 21 jul. 2016.

FRANCISCO, P.M.S.B.; DONALISIO, M.R.; GABRIEL, F.J.O.; BARROS, M.B.A. Hepatitis B vaccination in adolescents living in Campinas, São Paulo, Brazil. Rev. bras. epidemiol., São Paulo, v. 18, n. 3, p. 552-567, set. 2015. Disponível em: <http://www.scielo.br/scielo.php?script=sci_arttext\&pid=S1415-790X2015000300552 \&lng=en\&nrm=iso>. Acesso em: 21 jul. 2016.

FREITAS, K.R.; DIAS, S.M.Z. Percepções de adolescentes sobre sua sexualidade. Texto contexto - enferm., Florianópolis , v. 19, n. 2, p. 351-357, jun. 2010 . Disponível em: <http://www.scielo.br/scielo.php?script=sci_arttext\&pid=S010407072010000200 $017 \& \operatorname{lng}=$ pt\&nrm=iso $>$. Acesso em: 20 jul. 2016.

GAZZINELLI, M.F.; SOUZA, V.; ARAÚJO, L.H.L.; COSTA, R.M.; SOARES, A.N.; MAIA, C.P.C. Teatro na educação de crianças e adolescentes participantes de ensaio clínico. Rev. Saúde Pública, São Paulo, v. 46, n. 6, p. 999-1006, dez. 2012. Disponível em: <http://www.scielosp.org/scielo.php?script=sci_arttext\&pid=S0034-891020120006 00009\&lng=pt\&nrm=iso $>$. Acesso em: 03 ago. 2016.

GERHARDT, T.E.; SILVEIRA, D.T. Métodos de pesquisa. Porto Alegre: Editora da UFRGS, 2009.

GONÇALVES, M.A.S. Construção da identidade moral e práticas educativas. Campinas: Papirus, 2015.

GREGO, M.C.; OHARA, C.V.S.; PEREIRA, S.R.; BRÊTAS, J.R.S. Oficina de autoexame de mamas: uma estratégia para o autoconhecimento de adolescentes. Acta paul. enferm., São Paulo, v. 24, n. 4, p. 493-499, 2011. Disponível em: $<$ http://www.scielo.br/scielo.php?script=sci_arttext\&pid=S0103-210020110004 00008\&lng=pt\&nrm=iso>. Acessos em: 30 jul. 2016.

HORTA, N.C.; SENA, R.R. The everyday health of the young individuals of a popular neighborhood of Belo Horizonte, Minas Gerais, Brazil. Rev. esc. enferm. USP, São Paulo, v. 45, n. spe2, p. 1673-1678, dez. 2011. Disponível em: <http://www.scielo.br/ scielo.php?script=sci_arttext\&pid=S0080-62342011000800006\&lng=pt\&nrm=iso $>$. Acesso em: 21 jul. 2016.

JESUS, M. C. P. O significado da educação sexual na relação pais/adolescentes. Rev. bras. enferm., Brasília, v. 52, n. 3, p. 455-468, set. 1999. Disponível em: 
$<$ http://www.scielo.br/scielo.php?script=sci_arttext\&pid=S0034-71671999000300015\& $\operatorname{lng}=$ pt\&nrm=iso $>$. Acessos em: 20 jul. 2016.

LACERDA, A.B.M.; SOARES, V.M.N.; GONCALVES, C.G.O.; LOPES, F.C.; TESTONI, R. Educational workshops as a strategy to promote hearing heath of adolescents: an exploratory study. Audiol., Commun. Res., São Paulo, v. 18, n. 2, p. 85-92, jun. 2013. Disponível em: <http://www.scielo.br/scielo.php?script=sci_arttext \&pid=S2317-64312013000200006\&lng=pt\&nrm=iso > . Acesso em: 29 jul. 2016.

MARINHO, J.C.B. Os modos de estruturação da Educação em Saúde na escola: das concepções e do currículo às práticas educativas e à aprendizagem. Dissertação (Mestrado). Programa de Pós-Graduação em Educação em Ciências: Química da Vida e Saúde, Universidade Federal do Rio Grande-FURG. Rio Grande, 2013. Disponível em: < http://repositorio.furg.br/handle/1/4797>. Acesso em: 20 jul. 2016.

MARINHO, J.C.B.; TAUCHEN, G.; SILVA, J.A. A percepção paradigmática sobre saúde presente nos trabalhos desenvolvidos com a temática educação em saúde na escola. In: III Encontro Nacional de Ensino de Ciências da Saúde e do Ambiente, 2012, Niterói. Anais... Niterói: UFF, 2012. Disponível em: <http://ivenecienciassubmissao. uff.br/index.php/ivenecienciassubmissao/eneciencias2012/paper/view/320/191>. Acesso em: 20 jul. 2016.

MARINHO, J.C.B.; SILVA, J.A.; CAETANO, M.R.V. Dilemas morais de saúde como estratégia de ensino para adolescentes. Enseñanza de las Ciencias, In press., 2017.

MENDES, L.R.; TEIXEIRA, M.L.O.; FERREIRA, M.A. Bebida alcohólica en la adolescencia: el cuidado-educación como estrategia de acción de la enfermería. Esc. Anna Nery, Rio de Janeiro, v. 14, n. 1, p. 158-164, mar. 2010. Disponível em: $<$ http://www.scielo.br/scielo.php?script=sci_arttext\&pid=S1414-81452010000100023 \&lng=es\&nrm=iso>. Acesso em: 21 jul. 2016.

NEVES, K.C.; TEIXEIRA, M.L.O.; FERREIRA, M.A. Fatores e motivação para o consumo de bebidas alcoólicas na adolescência. Esc. Anna Nery, Rio de Janeiro, v. 19, n. 2, p. 286-291, jun. 2015. Disponível em: <http://www.scielo.br/scielo.php?script =sci_arttext\&pid=S1414-81452015000200286\&lng=es\&nrm=iso $>$. Acesso em: 03 ago. 2016.

PRODANOV, C.C.; FREITAS, E.C. Metodologia do trabalho científico: métodos e técnicas da pesquisa e do trabalho acadêmico. 2 ed. Novo Hamburgo: Feevale, 2013.

RODRIGUES, E.M.; BOOG, M.C.F. Problematização como estratégia de educação nutricional com adolescentes obesos. Cad. Saúde Pública, Rio de Janeiro, v. 22, n. 5, p. 923-931, maio 2006. Disponível em: <http://www.scielo.br/scielo.php?script=sci_ arttext\&pid=S0102-311X2006000500005\&lng=pt\&nrm=iso>. Acesso em: 30 jul. 2016.

RUZANY, M.H. Atenção à Saúde do Adolescente: Mudança de Paradigma. In: BRASIL. Ministério da Saúde. Secretaria de Atenção à Saúde. Departamento de Ações Programáticas Estratégicas. Saúde do adolescente: competências e habilidades. Brasília: Editora do Ministério da Saúde, 2008. 
SANAEINASAB, H.; SAFFARI, M.; PAKPOUR, A.H.; NAZERI, M.; PIPER, C.N. A model-based educational intervention to increase physical activity among Iranian adolescents. J. Pediatr. (Rio J.), Porto Alegre, v. 88, n. 5, p. 430-438, out. 2012. Disponível em: <http://www.scielo.br/scielo.php?script=sci_arttext\&pid=S002175572012000500013\&lng=en\&nrm=iso>. Acesso em: 30 jul. 2016.

SANTANA, B.A.; ALVARENGA, K.F.; CRUZ, P.C.; QUADROS, I.A.; JACOBCORTELETTI, L.C.B. Prevention in a school environment of hearing loss due to leisure noise. Audiol., Commun. Res., São Paulo, v. 21, 2016. Disponível em: $<$ http://www.scielo.br/scielo. php?script=sci_arttext\&pid=S2317-64312016000100309 \&lng=pt\&nrm=iso>. Acesso em: 21 jul. 2016.

SANTOS, P.N.M.; ELIEL, R.A.; ELIEL, O. A Ciência e o novo estado do conhecimento: a contribuição da Ciência da Informação. Enc. Bibli: R. Eletr. Bibliocon. Ci. Inf., Florianópolis, n. 22, 2006.

SCHALL, V.T.; STRUCHINER, M. Educação em Saúde: novas perspectivas. Cad. Saúde Pública, Rio de Janeiro, v. 15, supl. 2, p. S4-SB6, 1999.

SILVA, K.L.; DIAS, F.L.A.; VIEIRA, N.F.C.; PINHEIRO, P.N.C. Reflexões acerca do abuso de drogas e da violência na adolescência. Esc. Anna Nery, Rio de Janeiro, v. 14, n. 3, p. 605-610, set. 2010. Disponível em: <http://www.scielo.br/scielo.php?script= sci_arttext\&pid=S1414-81452010000300024\&lng=pt\&nrm=iso >. Acesso em: 30 jul. 2016.

SILVA, A.R.V.; ZANETTI, M.L.; FORTI, A.C.; FREITAS, R.W.J.F.; HISSA, M.N.; DAMASCENO, M.M.C. Avaliação de duas intervenções educativas para a prevenção do Diabetes Mellitus tipo 2 em adolescentes. Texto contexto - enferm., Florianópolis, v. 20, n. 4, p. 782-787, dez. 2011. Disponível em: <http://www.scielo.br/scielo.php? script=sci_arttext\&pid=S0104-07072011000400018\&lng=pt\&nrm=iso $>$. Acesso em: 30 jul. 2016.

SILVA, J.G.; TEIXEIRA, M.L.O.; FERREIRA, M.A. Alimentação e saúde: sentidos atribuídos por adolescentes. Esc. Anna Nery, Rio de Janeiro, v. 16, n. 1, p. 88-95, mar. 2012. Disponível em: <http://www.scielo.br/scielo.php?script=sci_arttext\&pid=S141481452012000100012\& lng=pt\&nrm=iso>. Acesso em: 21 jul. 2016.

SILVA, D.C.A.; FRAZÃO, I.S.; OSORIO, M.M.; VASCONCELOS, M.G.L. Perception of adolescents on healthy eating. Ciênc. saúde coletiva, Rio de Janeiro, v. 20, n. 11, p. 3299-3308, nov. 2015. Disponível em: <http://www.scielo.br/scielo.php? script=sci_arttext\&pid=S1413-81232015001103299\&lng=pt\&nrm=iso >. acesso em: 21 jul. 2016.

SOARES, S.M.; AMARAL, M.A.; SILVA, L.B.; SILVA, P.A.B. Oficinas sobre sexualidade na adolescência: revelando vozes, desvelando olhares de estudantes do ensino médio. Esc. Anna Nery, Rio de Janeiro, v. 12, n. 3, p. 485-491, set. 2008. Disponível em: <http://www.scielo.br/ scielo.php?script=sci_arttext\&pid=S141481452008000300014\&lng=en\&nrm=iso>. Acesso em: 20 jul. 2016. 
TOGNETTA, L.R.P.; ASSIS, O.Z.M. A construção da solidariedade na escola: as virtudes, a razão e a afetividade. Educação e Pesquisa, São Paulo, v.32, n.1, p. 49-66, jan./abr. 2006. Disponível em: <http://www.revistas.usp.br/ep/article/ view/27997/29784 >. Acesso em: 13 abr. 2014.

VIERO, V.S.F.; FARIAS, J.M.; FERRAZ, F.; SIMÕES, P.W.; MARTINS, J.A.; CERETTA, L.B. Educação em saúde com adolescentes: análise da aquisição de conhecimentos sobre temas de saúde. Esc. Anna Nery, Rio de Janeiro, v. 19, n. 3, p. 484-490, set. 2015. Disponível em: <http://www.scielo.br/scielo.php?script=sci_arttext \&pid=S1414-81452015000300484\&lng=en\&nrm=iso>. Acesso em: 21 jul. 2016. 